\title{
Agnès Roche. Des vies de pauvres. Les classes populaires dans le monde rural
}

Rennes, Presses universitaires de Rennes, 2016, 340 p.

\section{Florent Bidaud}

\section{(2) OpenEdition}

\section{Journals}

Édition électronique

URL : http://journals.openedition.org/economierurale/5292

DOI : $10.4000 /$ economierurale.5292

ISSN : 2105-2581

\section{Éditeur}

Société Française d'Économie Rurale (SFER)

Édition imprimée

Date de publication : 15 octobre 2017

Pagination : 93-96

ISSN : 0013-0559

Référence électronique

Florent Bidaud, "Agnès Roche. Des vies de pauvres. Les classes populaires dans le monde rural », Économie rurale [En ligne], 361 | septembre-octobre, mis en ligne le 15 octobre 2017, consulté le 23 septembre 2020. URL : http://journals.openedition.org/economierurale/5292 ; DOI : https://doi.org/ 10.4000/economierurale.5292

Ce document a été généré automatiquement le 23 septembre 2020.

(c) Tous droits réservés 


\title{
Agnès Roche. Des vies de pauvres. Les classes populaires dans le monde rural
}

Rennes, Presses universitaires de Rennes, 2016, 340 p.

\author{
Florent Bidaud
}

\section{RÉFÉRENCE}

Agnès Roche. Des vies de pauvres. Les classes populaires dans le monde rural. Rennes, Presses universitaires de Rennes, 2016, 340 p.

1 Issu d'une recherche sur la pauvreté menée de 2011 à 2013 dans les campagnes du Puyde-Dôme, Des vies de pauvres s'inscrit dans une lignée d'ouvrages qui, depuis les Chômeurs de Marienthal ${ }^{1}$, a pris pour objet le quotidien des milieux populaires en épousant le point de vue des travailleurs sociaux. L'observation d'interactions avec les assistantes sociales du Conseil général et de la MSA a en effet servi de porte d'entrée pour interroger des usagers réguliers des services sociaux. Suivant la méthode des récits de vie, 110 personnes ont été longuement interviewées, hommes et femmes à parité - 12 « jeunes relégués », 21 "retraités modestes », 33 «travailleurs pauvres en galère et éclopés de la vie » et 44 «paysans »-, dans quatre territoires aux profils contrastés, plus ou moins pauvres ou "attractifs ", plutôt orientés vers l'élevage ou vers les grandes cultures (Livardois, Combrailles, Sancy et Limagne). Le guide d'entretien visait à aborder différents thèmes, sans ordre préconçu: les parents et grands-parents, le parcours scolaire et professionnel, la situation matrimoniale, les ressources du ménage, etc.

2 L'ouvrage se veut un back to basics de la sociologie des classes sociales. C'est le cas pour la définition monétaire de la pauvreté posée dès l'introduction, bien ajustée à l'entrée par les dispositifs d'aide sociale. « Être pauvre, c'est avoir peu » et les classes de revenu dessinent, par paliers, des classes ou des catégories sociales bien distinctes (classes 
populaires, classes moyennes et catégories aisées). L'auteure entend enquêter non sur la "misère de position », relative, « du point de vue de celui qui la vit », comme l'avait fait avec une certaine sophistication l'équipe de Pierre Bourdieu dans La misère du monde $e^{2}$, mais sur "la misère de condition ", «la grande misère », celle vécue au quotidien par les « fractions les plus pauvres des classes populaires, précaires ou non ». De même, elle dénonce les écrans de fumée que forment de nouvelles catégories administratives, par exemple le nouveau zonage en aires urbaines de l'INSEE, «qui a pour effet de faire disparaître l'espace rural », alors qu'en retenant l'ancien zonage, « la population rurale est de 13,9 millions d'habitants au recensement de 2007, en augmentation de $9 \%$ depuis le recensement de 1999 ».

3 La première partie réorganise le matériau recueilli en une centaine de courts portraits qui «essaient de rendre compte de l'histoire de la personne et de sa situation présente", en recherchant "la variété des individus, des situations et des trajectoires ». Chaque texte se lit rapidement et fournit une variation par rapport aux cas voisins. Le portrait basé sur un récit de vie permet de restituer, de l'intérieur, des existences et des trajectoires à la fois précaires et surdéterminées par leurs conditions sociales. Il ne s'agit pas de retranscriptions verbatim, mais le lecteur accède tout de même à de larges extraits des propos recueillis. La seconde partie, bien plus courte, dégage des grilles de lecture et établit des ponts entre les éléments présentés dans la première partie et d'autres travaux menés, en France, sur les classes populaires. Elle revient ainsi sur les différentes catégories (jeunes, retraités, précaires, paysans), avant d'analyser transversalement " les mécanismes de la domination et de la reproduction ».

4 Les portraits des "jeunes relégués » donnent souvent à voir des enfances difficiles, exposées à une violence crue (viols, passages à tabac) ou tournée vers soi-même (automutilations, alcoolisme et addictions, etc.). Ces jeunes se mettent en couple et ont des enfants très tôt, et «les problèmes semblent déjà se reproduire ». L'entrée dans l'âge adulte se fait sans grande motivation, que ce soit dans le domaine des études ou du travail. «On imagine mal comment » la douzaine de jeunes interviewés « va pouvoir se sortir de la précarité banalisée ». De même, pour les travailleurs pauvres, "éclopés de la vie ", atteints par une maladie grave et handicapés, dépressifs, dépendants de l'alcool ou des drogues, etc. Avec les retraités pauvres, "pauvres devenus vieux », «la lecture des transcriptions d'entretien laisse une impression de résignation, d'acceptation du destin, et les moments heureux semblent absents ».

5 Dans cette litanie de misères, le chapitre consacré aux portraits d'agriculteurs, intitulé "La fin des petits paysans ", est sans doute le plus équilibré et le plus intéressant. Il est aussi le seul à comporter une typologie, établie à partir de "deux points capitaux " explicités dans la deuxième partie de l'ouvrage : l'héritage et la modernisation. D'une part, « hériter de beaucoup, ou de peu, voire de rien [...] est un élément qui oriente la suite de l'histoire d'un paysan ». D'autre part, s'intéresser à la façon dont celui-ci « envisage et réalise concrètement la modernisation de son exploitation » permet de saisir des dimensions plus subjectives, telles que la motivation et l'intérêt pour le travail, les aspirations, les choix d'investissements, etc.

6 Le premier sous-groupe est celui des producteurs " en sursis ", qui "vivotent sur de petites exploitations", "héritiers vieillissants, condamnés à disparaître ", sans repreneur dans la famille. Tel est le cas de Louis, 57 ans, 7 hectares, des problèmes de santé, et célibataire. Ou de Laurence, installée dans les années 1990 sur 10 hectares de Limagne hérités par son mari. Cet apport n'est pas suffisant et les terres sont trop 
chères, dans la région, pour atteindre une taille critique en le complétant par la location. Deuxième catégorie, les « agriculteurs en survie » ont hérité d'exploitations de taille moyenne, difficiles à moderniser. Ils font des choix discutables, n'investissent pas sur du matériel de traite, par exemple, ou au contraire se suréquipent en espérant rester compétitifs. Un « grain de sable » peut venir gripper leur trajectoire déjà fragile : problème de santé, placements peu judicieux, etc. Enfin, un troisième profil est constitué par des "non-héritiers, souvent militants qui ont choisi d'être paysan». Ceux-ci «ont parfois mal estimé les chances de succès de leur entrée dans l'agriculture ", et comme les autres, peuvent en concevoir certaines rancœurs contre la PAC, les SAFER, les syndicats agricoles, etc., qu'ils tiennent pour responsables, au moins en partie, de leurs difficultés.

7 Cette tripartition est suggestive. Vu l'effort nécessaire pour réaliser de nombreux entretiens approfondis, on peut regretter que l'auteure n'ait pas complété son tableau en interrogeant d'autres agriculteurs (ceux qui ne se présentent pas aux guichets sociaux), et ce d'autant plus qu'elle souligne la continuité des situations entre les in et les out. Mais à quoi bon? «Beaucoup d'agriculteurs ne comprennent rien au système agricole et se sentent complètement dépassés. » Les mécanismes de renouvellement (et d'éviction des exploitations en dessous d'un certain seuil de viabilité) opèrent à un autre niveau, supra-individuel et macro-historique, que seules des méthodes recourant aux statistiques et à la modélisation peuvent éclairer ${ }^{3}$. Avec le regain d'intérêt pour l'agriculture et les changements de pratiques ${ }^{4}$, on voit par ailleurs fleurir des sociologies du travail combinant portraits d'individus et élaboration de types sociaux ${ }^{5}$, et il serait intéressant de discuter les recoupements et le caractère cumulatif (ou pas) de ces différents travaux.

Plus généralement, le choix de mettre ainsi en valeur des récits de vie à l'exclusion de tout autre matériau est à double tranchant. D'un côté, une certaine qualité esthétique de l'ouvrage, qui voudrait «dessiner une fresque du monde populaire dans les campagnes ", "à la manière dont Brueghel l'Ancien a peint ses personnages au XVI ${ }^{e}$ siècle ", "une fresque vivante, où s'animent des dizaines de personnages", et qui évoque aussi Zola et Hugo-tout en prenant ses distances avec l'écueil du " misérabilisme ». Mais à rebours de cette intention, l'accumulation d'anecdotes sur des personnes "ordinaires", aux destins contrariés ou brisés, pourra aussi irriter le lecteur, gagné par l'impression de faire du surplace pendant 200 pages.

L'auteure ne prétend pas que les récits de vie donnent un accès direct aux conditions d'existence des personnes interviewées. Mais les portraits produisent une certaine clôture de l'analyse sur le corpus de textes constitué. Quand elle essaie d'en sortir pour analyser la contribution des institutions (école, famille, travail social) aux situations de domination et à la reproduction, Agnès Roche généralise surtout en faisant référence aux travaux d'autres sociologues, équipés pour des traitements quantitatifs et étudiant d'autres terrains que la pauvreté en zones rurales. L'école apparaît ainsi comme un lieu, sinon un outil, de «tri social » où l'orientation en filière professionnelle s'opère « naturellement » mais efficacement, à coup de violences symboliques. De son côté, la famille paraît être un facteur de dérèglement des trajectoires, mais aussi un filet de sécurité, grâce aux relations d'entraide.

10 Ce type de constats et d'analyses mérite sans doute d'être réitéré, mis à l'épreuve des nouvelles conditions sociales (multiplication des SDF dans les années 1990, ou actuellement, débat sur la relégation dans une « France périphérique »), etc., mais il est 
aujourd'hui très familier. S'agissant de son ambition de mieux connaître les milieux populaires en zones rurales, on peut donc penser que le livre tient surtout ses promesses dans sa partie documentaire, même si elle est apparemment la plus anecdotique.

Parler de "classes populaires » ne va pas de soi. L'auteure cite à ce propos Olivier Schwartz, soulignant qu'«il est absolument nécessaire que la recherche montre “empiriquement que les sujets qu'elle étudie sont effectivement placés dans des conditions économiques et sociales dominées, et que leurs modes de vie, leur manière d'être révèlent des formes de séparation culturelle" ». Au-delà de la mise en lumière de situations de décrochage et de relégation dans un état stationnaire, irrémédiable, qu'on peut éventuellement qualifier de "misère de condition", l'ouvrage ne semble pas apporter une démonstration de ce type. Dans les trajectoires esquissées par les portraits de pauvres, le hasard des rencontres, des problèmes de santé, les goûts et dégoûts personnels (pour les études, le travail, etc.) semblent jouer un rôle plus important que la position dans les rapports de production. De plus, "la dimension culturelle de la pauvreté n'a pas été abordée » et l'ouvrage ne dégage pas clairement des styles de vie ou un habitus de classe lié à cette position fantôme dans les rapports sociaux ${ }^{6}$. Là encore, il aurait été utile d'ouvrir l'enquête au-delà de l'échantillon spontané constitué par les guichets sociaux.

Malgré ses limites assumées, l'auteure pointant différents «angles morts » de son projet, Des vies de pauvres constitue une contribution intéressante à la sociologie rurale. L'introduction souligne à juste titre la rareté des travaux sur un objet plus difficile à construire sociologiquement que l'étude de la relégation dans les concentrations urbaines. Sa partie documentaire pourra également nourrir le débat sur les politiques publiques (éducation nationale, aides sociales et logement, PAC et développement rural, etc.), en donnant accès à des réalités souvent méconnues des décideurs publics, généralement issus de milieux plus favorisés. En résumé, cet ouvrage a le mérite de révéler la part souvent invisible de la France des campagnes.

\section{NOTES}

1. Lazarsfeld P., Jahoda M., Zeisel H. (1982). Les chômeurs de Marienthal. Paris, Minuit (1re éd. 1931).

2. Bourdieu P. (dir.) (1993). La misère du monde. Paris, Seuil.

3. Mazoyer M., Roudart L. (1997). Histoire des agricultures du monde. Du néolithique à la crise contemporaine. Paris, Seuil.

4. Lamy J. (2016). Le grand remembrement. La sociologie des savoirs ruraux depuis les années 1950. Zilsel, n 1, pp. 263-291.

5. Par exemple, Samak M. (2016). La politisation variable des alternatives agricoles. Savoir/Agir, $\mathrm{n}^{\circ} 38$, pp. 29-35, sur les néo-fermiers permaculteurs ; Nicolas F. (2017). L'agriculture biologique : un travail pas comme les autres? Tracés, $\mathrm{n}^{\circ} 32$, pp. 69-90.

6. Sur la définition des classes sociales et les habitus de classe, voir notamment Bourdieu P. (1979). La distinction. Critique sociale du jugement. Paris, Minuit. 


\section{AUTEUR}

\section{FLORENT BIDAUD}

Centre d'études et de prospective Ministère de l'Agriculture et de l'Alimentation 